University of Minnesota Morris Digital Well

University of Minnesota Morris Digital Well

Mathematics Publications

Faculty and Staff Scholarship

2019

\title{
L-series and Feynman Integrals
}

David Broadhurst

Open University

David P. Roberts

University of Minnesota - Morris, roberts@morris.umn.edu

Follow this and additional works at: https://digitalcommons.morris.umn.edu/mathematics

Part of the Mathematics Commons

\section{Recommended Citation}

David Broadhurst and David P. Roberts. L-Series and Feynman Integrals. 2017 MATRIX Annals (2019), 401-403.

This Article is brought to you for free and open access by the Faculty and Staff Scholarship at University of Minnesota Morris Digital Well. It has been accepted for inclusion in Mathematics Publications by an authorized administrator of University of Minnesota Morris Digital Well. For more information, please contact skulann@morris.umn.edu. 


\title{
L-series and Feynman Integrals
}

\author{
David Broadhurst and David P. Roberts
}

\begin{abstract}
Integrals from Feynman diagrams with massive particles soon outgrow polylogarithms. We consider the simplest situation in which this occurs, namely for diagrams with two vertices in two space-time dimensions, with scalar particles of unit mass. These comprise vacuum diagrams, on-shell sunrise diagrams and diagrams obtained from the latter by cutting internal lines. In all these cases, the Feynman integral is a moment of $n=a+b$ Bessel functions, of the form $M(a, b, c):=\int_{0}^{\infty} I_{0}^{a}(t) K_{0}^{b}(t) t^{c} \mathrm{~d} t$. The corresponding L-series are built from Kloosterman sums over finite fields. Prior to the Creswick conference, the first author obtained empirical relations between special values of L-series and Feynman integrals with up to $n=8$ Bessel functions. At the conference, the second author indicated how to extend these. Working together we obtained empirical relations involving Feynman integrals with up to 24 Bessel functions, from sunrise diagrams with up to 22 loops. We have related results for moments that lie beyond quantum field theory.
\end{abstract}

\section{Physical and mathematical context}

The context for our work is given in [1]. At the conference, the first author reported on the magnificent progress made by Stefano Laporta, whose solitary decade-long effort on the magnetic moment of the electron has come to fruition [4]. This involves, inter alia, moments of 6 Bessel functions, one of which

$$
M(1,5,1):=\int_{0}^{\infty} I_{0}(t) K_{0}^{5}(t) t \mathrm{~d} t=\frac{\pi^{2} L_{6}(2)}{2}
$$

David Broadhurst

Open University, UK, e-mail: David.Broadhurst@open.ac.uk

David P. Roberts

University of Minnesota Morris, USA, e-mail: roberts@morris.umn.edu 
is empirically related in [1] to the central value of the L-series of modular weight 4 and conductor 6, with Fourier series $q \prod_{k>0}\left(1-q^{k}\right)^{2}\left(1-q^{2 k}\right)^{2}\left(1-q^{3 k}\right)^{2}\left(1-q^{6 k}\right)^{2}=$ $\sum_{k>0} A_{6}(k) q^{k}$. This modular form also delivers the Bessel moments

$$
\begin{aligned}
M(2,4,1) & :=\int_{0}^{\infty} I_{0}^{2}(t) K_{0}^{4}(t) t \mathrm{~d} t=\frac{3 L_{6}(3)}{2} \\
M(3,3,1) & :=\int_{0}^{\infty} I_{0}^{3}(t) K_{0}^{3}(t) t \mathrm{~d} t=\frac{3 L_{6}(2)}{2} .
\end{aligned}
$$

For $n=8$ Bessel functions, the L-series of a modular form of weight 6 and conductor 6 likewise gives evaluations of $M(1,7,1), M(2,6,1), M(3,5,1)$ and $M(4,4,1)$. The challenge presented at the conference was to find comparable relations between Lseries and moments of $n>8$ Bessel functions.

\section{Progress at Creswick}

This challenge was met by considering determinants of Feynman integrals, by allowing for adjustment of the local Kloosterman data, at primes that divide the conductor, and by empirical determination of the conductor, sign and gamma factors that enter the functional equation for the L-series. The good factors are classical and much useful information towards conductors was available in [5]. The formalism of [2] pointed us to the correct determinants. Using the methods in [3] for numerical computation of L-series, we were able to progress beyond the modular forms studied in [1].

We were successful for odd Bessel numbers up to $n=17$ and even Bessel numbers up to $n=20$. Let $\Omega_{a, b}$ be the determinant of the $r \times r$ matrix with $M(a, b, 1)$ at top left, size $r=\lceil(a+b) / 4-1\rceil$, powers of $t^{2}$ increasing to the right and powers of $I_{0}^{2}(t)$ increasing downwards. Then

$$
\begin{aligned}
L_{17}(8) & =\frac{2^{15} \times 29 \Omega_{2,15}}{3^{5} \times 5^{2} \times 7 \pi^{12}} \\
L_{20}(10) & =\frac{2^{12} \times 11 \times 131 \Omega_{1,19}}{3^{11} \times 5^{6} \times 7^{3} \pi^{20}} \\
L_{20}(11) & =\frac{2^{19} \times 17 \times 19 \times 23 \Omega_{2,18}}{3^{13} \times 5^{7} \times 7^{3} \pi^{12}}
\end{aligned}
$$

are among findings made and presented at the conference.

\section{Subsequent progress}

From data on Kloosterman sums in finite fields $\mathbf{F}_{q}$ with $q<200000$, we found 


$$
\begin{aligned}
L_{19}(8) & =\frac{2^{14} \times 1093 \times 13171 \Omega_{2,17}}{3^{4} \times 5^{4} \times 7 \times 11 \pi^{20}} \\
L_{24}(12) & =\frac{2^{29} \times 12558877 \Omega_{1,23}}{3^{19} \times 5^{9} \times 7^{3} \times 11 \pi^{30}} \\
L_{24}(13) & =\frac{2^{27} \times 17 \times 19^{2} \times 23^{2} \times 46681 \Omega_{2,22}}{3^{23} \times 5^{12} \times 7^{4} \times 11^{2} \pi^{20}} .
\end{aligned}
$$

In parallel with the above results for odd moments, we obtained relations between even moments of Bessel functions and L-series determined by a quadratic twist of Kloosterman data. We have conjecturally complete sets of quadratic relations for both types of moment, encoded by Betti and de Rham matrices, for which we provide explicit constructions. In some cases where the sign of the functional equation is odd, we are able to define regulated moments that deliver central derivatives.

Acknowledgements We thank Ling Long, Masha Vlasenko and Wadim Zudilin for their splendid organization of a three-week conference on Hypergeometric motives and Calabi-Yau differential equations, held at Creswick, Australia, in January 2017, and the MATRIX Institute for financial support. DB thanks the University of Newcastle, NSW, and the Mainz Institute for Theoretical Physics, for hospitality and support that enabled further progress. DPR's research is supported by grant DMS-1601350 from the National Science Foundation.

\section{References}

1. Broadhurst, D: Feynman integrals, L-series and Kloosterman moments. Communications in Number Theory and Physics 10, 527-569 (2016), http://arxiv.org/abs/1604.03057

2. Deligne, P: Valeurs de fonctions $L$ et périodes d'intégrales. Proceedings of Symposia in Pure Mathematics 33, 313-346 (1979), http://publications.ias.edu/deligne/paper/379

3. Dokchitser, T: Computing special values of motivic L-functions. Experimental Mathematics 13, 137-149 (2004), http://arxiv.org/abs/math/0207280

4. Laporta, S: High-precision calculation of the 4-loop contribution to the electron $g-2$ in QED, http://arxiv.org/abs/1704.06996

5. Yun, Zhiwei: Galois representations attached to moments of Kloosterman sums and conjectures of Evans. Compositio Mathematica 151, 68-120 (2015), http://arxiv.org/abs/1308.3920 